\title{
Broad-spectrum Tuning of Surface Plasmon Resonance Using Palladium Nanorods
}

\author{
Chunyu Chen ${ }^{1,2}$, Jun Wang ${ }^{1}$, Yachen $\mathrm{Gao}^{1^{*}}$ \\ ${ }^{1}$ College of Electronic Engineering, Heilongjiang University, Harbin 150080, China \\ ${ }^{2}$ School of Mechatronics Engineering, Daqing Normal University, Daqing 163712, China
}

Corresponding Author Email: gaoyachen@hlju.edu.cn

https://doi.org/10.18280/acsm.430406

Received: 7 March 2019

Accepted: 10 June 2019

\section{Keywords:}

palladium nanorods, absorption spectra, surface plasmon resonance (SPR), finitedifference time-domain (FDTD) method

\begin{abstract}
This paper aims to achieve broad-spectrum tuning of surface plasmon resonance (SPR) with palladium nanorods. For this purpose, the finite-difference time-domain (FDTD) method was selected to simulate the optical properties of palladium nanorods. Specifically, we investigated the effects of radius, axial length, and aspect ratio of palladium nanorods on the SPR, the impacts of axial length on SPR of palladium and gold nanorods of the same size, and the influence of radius on palladium nanospheres and nanorods of the same axial length. The absorption spectra of palladium nanorods in different sizes were also analyzed. The results show that the longitudinal absorption peak of palladium nanorods can be used to tune the SPR from the visible region to the infrared region; palladium nanorods are more suitable for broad-spectrum tuning of the SPR than gold nanorods; palladium nanorods are more effective for broad-spectrum SPR tuning than palladium nanospheres; changing the size of palladium nanorods can effectively tune the SPR across a broad spectrum. The research findings shed important new light on the design of surface plasmon scales, filters, biosensors, etc.
\end{abstract}

\section{INTRODUCTION}

Metal nanoparticles can absorb the incident light of a certain wavelength. The obvious absorption band is corresponded to the ultraviolet-visible region. In this band, surface plasmon resonance (SPR) may occur, enhancing the local electric field. The SPR refers to the electromagnetic response that occurs when the free electrons are oscillating at the same frequency with the incident light on the surface of a material [1]. The SPR is typically observed on gold, which can be tuned from 523 to $1,550 \mathrm{~nm}$ [2-5]. However, the SPR tuning is severely lacking for non-gold applications.

Like gold, palladium is also a rare metal with obvious SPR effect. The absorption peaks of palladium nano-cubes are $212 \mathrm{~nm}$. The linear optical properties of palladium nanoparticles, ranging from extinction, absorption to scattering, depend heavily on their size and shape, as well as the surrounding media [6]. Many scholars have explored deep into palladium nanoparticles. For example, some scholars studied nano-cubes, triangular nano-disks and hexagonal nano-disks of palladium, and assembled palladium-core silver-shell nano-cubes [6-8]. some scholars prepare core-shell Au-Pd@Pd icosahedral nanoparticles with trapezoidal shell and core-shell Au@Pd nanorods [9, 10]. Mahmoud [11, 12] probed into the extinction features of gold nanocage structure, hollow core-shell structure of gold-palladium double shell, and hollow core-shell rattle structure of gold-palladium double shell. Some scholars investigate Pd concave nano-cubes, $\mathrm{M} @ \mathrm{Au}(\mathrm{M}=\mathrm{Pd}, \quad \mathrm{Cu}-\mathrm{Pt})$ mixed nano-stars, palladium nanosheets, $\mathrm{Au}$ nanospheres with core-shell structure, revealing that the SPR is tunable in dimer nano-antennas of transition metal (e.g. platinum and palladium) and palladiumsilver bimetallic nano-catalysts [13-18]. Kim et al. [19] prepared core-shell nanoparticles of double-shell (palladiumplatinum) structure for a 3D defocusing direction sensor. Wang et al. [20] prove that Au-Pd alloy and lactate dehydrogenase can work together and promote the selective oxidation of benzyl alcohol under photocatalysis. Nugroho et al. [21] establish the general scale and design rules for hydrogen-induced optical properties of palladium and palladium alloy nanoparticles. Wang et al. [22] grow palladium nanoparticles on polydopamine coated with $\mathrm{Fe}_{2} \mathrm{O}_{3}$, and use them as high performance recoverable nano catalysts. Watkins and Yves [23] fabricate ultra-sensitive and fast plasma hydrogen sensors with palladium nanofilms. Xu et al. [24] calculates the photothermal coupling factor based on palladium nanoparticles-loaded $\mathrm{TiO}_{2}$ to reduce $\mathrm{CO}_{2}$.

The above studies mainly focus on preparing palladium nanoparticles and applying them in such fields as catalysis, hydrogen production and sensor fabrication. However, there is little report on the implementation of palladium nanoparticles in broad-spectrum SPR tuning. To make up for this gap, this paper attempts to design a reasonable structure of palladium nanoparticles that can tune the SPR across broad spectrum. Hence, the optical properties of palladium nanorods were simulated by finite-difference time-domain (FDTD) method. Firstly, the author explored how the SPR is affected by radius, axial length, and aspect ratio, respectively. Next, the author analyzed the effect of axial length on SPR of palladium and gold nanorods of the same size, and then the effect of radius on palladium nanospheres and nanorods of the same axial length. Finally, the absorption spectra of palladium nanorods in different sizes were observed in details. The research aims to promote the preparation of palladium nanomaterials. 


\section{SIMULATION}

There are three popular numerical methods to analyze metal nanoparticles, namely, discrete dipole approximation (DDA), finite-element method (FEM) and finite-difference timedomain (FDTD) method. In this paper, the transmission properties of palladium nanoparticles are simulated by an FDTD software. During the simulation, the light was incident along the $\mathrm{z}$-axis, and polarized along the $\mathrm{x}$-axis. The periodic boundary condition (PBC) was adopted for both $\mathrm{x}$ - and $\mathrm{y}$-axes, and the perfectly matched layer (PML) boundary condition was selected for z-axis. The dielectric constant of palladium was obtained from the handbook authored by Edward D. Palik. The dielectric environment was configured as the air. The refractive index was set to 1.0. The FDTD model (Figure 1) includes a cylinder in the middle and two hemispheres at both ends, where $a$ refers to radius and $b$ represents axial length.

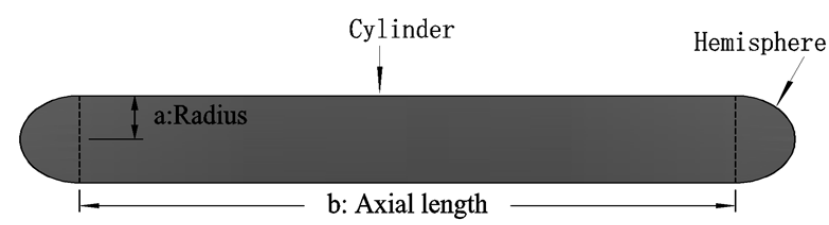

Figure 1. The FDTD model

\section{RESULTS AND DISCUSSION}

\subsection{Effects of radius on the SPR}

The absorption spectra of palladium nanorods with the same axial length $(54 \mathrm{~nm})$ and different radii $(5,6,8$ and $9 \mathrm{~nm})$ are displayed in Figure 2. It can be seen that each palladium nanorod had two absorption peaks, a transverse absorption peak and a longitudinal absorption peak. With the growth in radius, the wavelength of the transverse absorption peak shifted from 353 to $329 \mathrm{~nm}$, while the longitudinal absorption peak moved from 780 to $546 \mathrm{~nm}$.

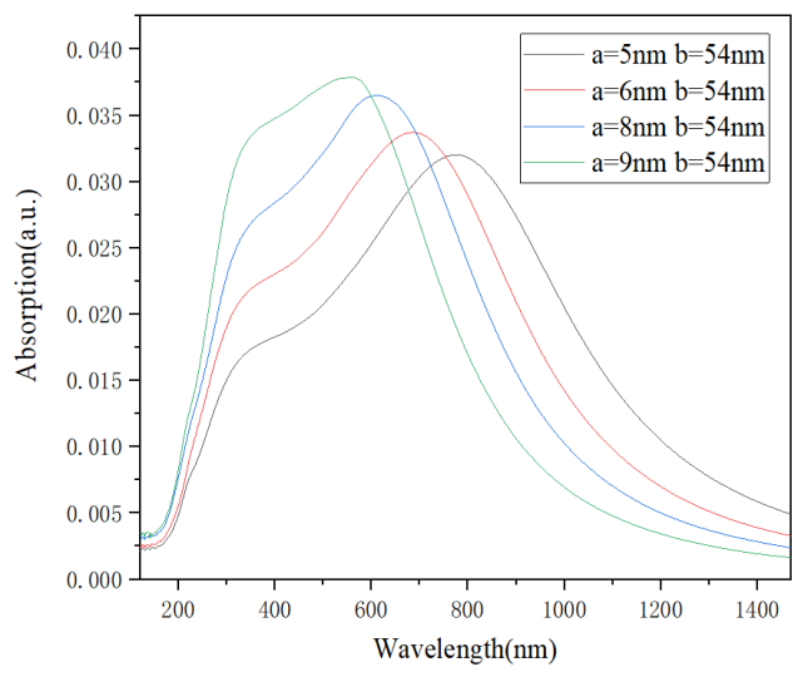

Figure 2. Absorption spectra of palladium nanorods with the same axial length and different radii

The relationship between each absorption peak and the radius is plotted as Figure 3. It can be observed that the longitudinal absorption peaks blue shifted faster and widened more obviously than the transverse absorption peaks.

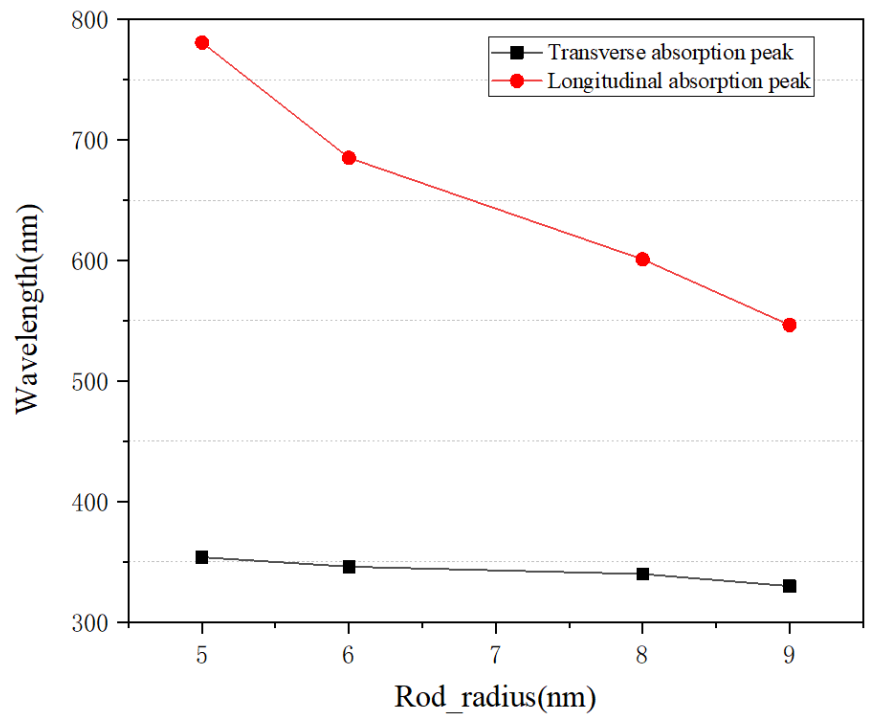

Figure 3. The relationship between each absorption peak and the radius

\subsection{Effects of axial length on the SPR}

The absorption spectra of palladium nanorods with the same radius $(6 \mathrm{~nm})$ and different axial lengths $(40,50,70,80,90$, $110,120,130,140,150,160,170$ and $180 \mathrm{~nm}$ ) are presented in Figure 4. It can be seen that, with the increase of axial length, both transverse and longitudinal absorption peaks of palladium nanorods were red shifted. The transverse absorption peak appeared near $340 \mathrm{~nm}$, and always red shifted slowly in the ultraviolet region. Meanwhile, the longitudinal absorption peak red shifted from the visible region $(597 \mathrm{~nm})$ to infrared region $(1,386 \mathrm{~nm})$. The longitudinal absorption peak red shifted faster and widened more obviously than the transverse absorption peak. With the transverse absorption peak in the ultraviolet region and the longitudinal absorption peak in visible-infrared region, the palladium nanorods have achieved broad-spectrum SPR, which is tunable in ultravioletvisible-infrared regions.

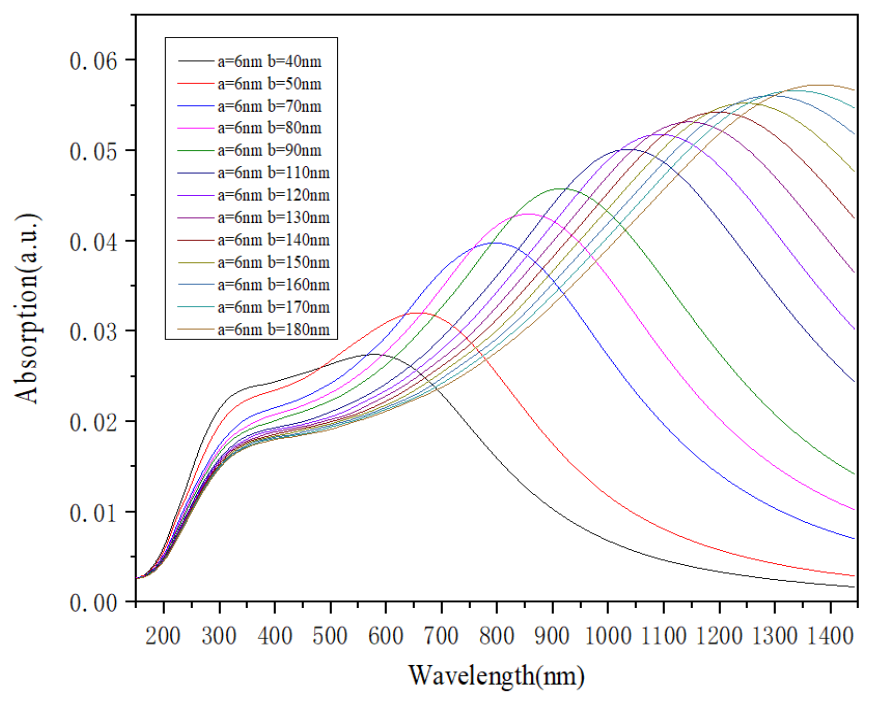

Figure 4. Absorption spectra of palladium nanorods with the same radius and different axial lengths

The relationship curve between the longitudinal absorption peak and the axial length is described in Figure 5. It can be 
learned that, as the axial length of palladium nanorods was increased continuously from 40 to $180 \mathrm{~nm}$, the longitudinal absorption peak saw a linear increase in wavelength, and surpassed the transverse absorption peak in red shift sensitivity. The slope $k$ of the relationship curve equals the ratio of the mean wavelength change $\Delta \lambda$ of the absorption peak to the change of axial length $\Delta b$ :

$$
k=\frac{\sum \Delta \lambda}{\sum \Delta b}=5.24
$$

After knowing the position of absorption peak, the longitudinal size of palladium rod can be estimated to save the cost of transmission electron microscopy (TEM). Moreover, the colloidal solution of palladium nanorod with extremely uniform morphology can be produced by precisely controlled synthesis and post-treatment. The palladium nanorods with minimal individual differences can be used as a standard reference at the nanoscale.

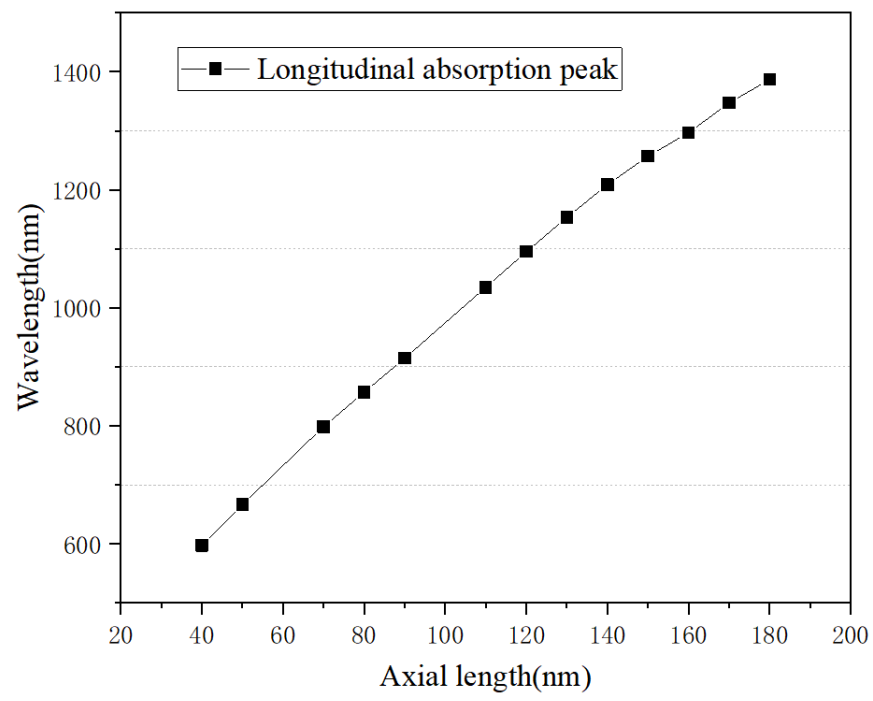

Figure 5. The relationship between longitudinal absorption peak and the axial length

\subsection{Effects of aspect ratio on the SPR}

The aspect ratio refers to the quotient of length and diameter. For palladium nanorods, the length equals the sum of diameter and axial length. Figure 6 presents the absorption spectra of several palladium nanorods, which differ in radii $(3,4,5,6,7$ and $8 \mathrm{~nm})$ and axial lengths $(54,72,90,108,126$ and 144nm) but agree in aspect ratio (10). As shown in the figure, with the increase in size, transverse absorption peak showed a small blue shift, the longitudinal absorption peak was red shifted, and the absorption rate was on the rise.

Similarly, Figure 7 shows the absorption spectra of several palladium nanorods, which also differ in radii $(2,3,4,5,6,7$ and 8nm) and axial lengths (60, 90, 120, 150, 180, 210 and $240 \mathrm{~nm}$ ) but agree in aspect ratio (16). Obviously, the transverse and longitudinal absorption peaks followed the same trend as in Figure 6.

The above two figures demonstrate that, when palladium nanorods have a fixed aspect ratio and an expanding size, the transverse absorption peak will blue shift slightly, the longitudinal absorption peak will be red-shifted, and the absorption rate will increase.

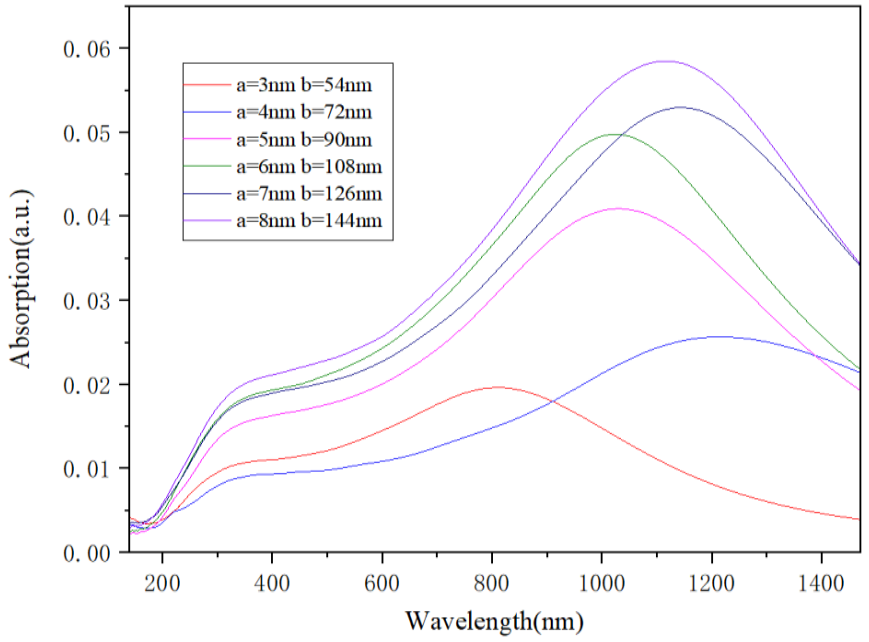

Figure 6. Absorption spectra of palladium nanorods with different sizes and the same aspect ratio (10)

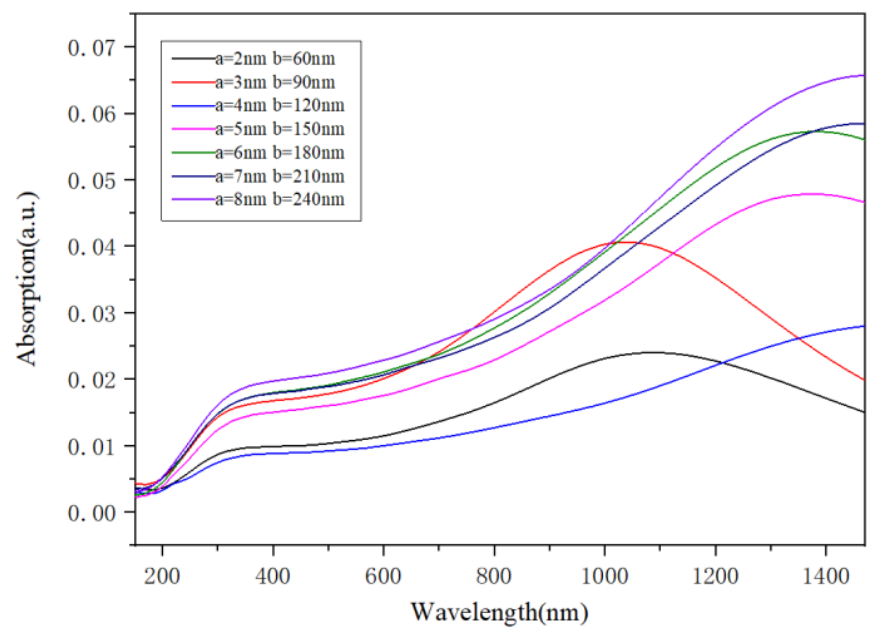

Figure 7. Absorption spectra of palladium nanorods with different sizes and the same aspect ratio (16)

\subsection{Effects of axial length on the SPR of palladium and gold nanorods of the same size}

Figure 8 shows the absorption spectra of gold nanorods with the same radius $(6 \mathrm{~nm})$ and different axial lengths $(40,50,60$ and $70 \mathrm{~nm}$ ). As shown in Figure 8, with the growth in axial length, transverse absorption peak of goal nanorod slowly red shifted away from 540nm, while the longitudinal absorption peak quickly shifted from 670 to $770 \mathrm{~nm}$. Compared with transverse absorption peak, longitudinal absorption peak red shifted rapidly and obviously widened.

Figure 9 displays the absorption spectra of palladium nanorods with the same radius $(6 \mathrm{~nm})$ and different axial lengths $(40,50,60$ and $70 \mathrm{~nm})$. It can be seen that, with the increase of axial length, the transverse absorption peak of palladium nanorods slowly red shifted away from $340 \mathrm{~nm}$, while the longitudinal absorption peak shifted rapidly from 590 to $800 \mathrm{~nm}$. The longitudinal absorption peak of palladium nanorods red shifted faster than their transverse absorption peak, and also faster than longitudinal absorption peak of gold nanorods. The full width at half maximum (FWHM) of palladium nanorods had much greater growth than that of gold nanorods. This is because palladium has a wider conduction band than gold. The electrons at different positions of the conduction band differ in periods of motion. 


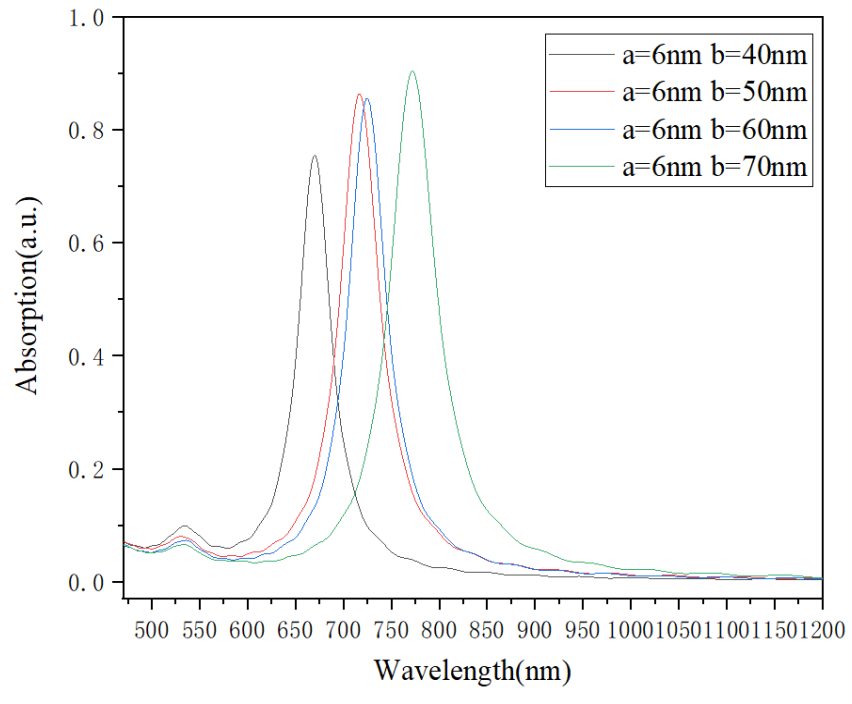

Figure 8. Absorption spectra of gold nanorods with different axial length and the same radius

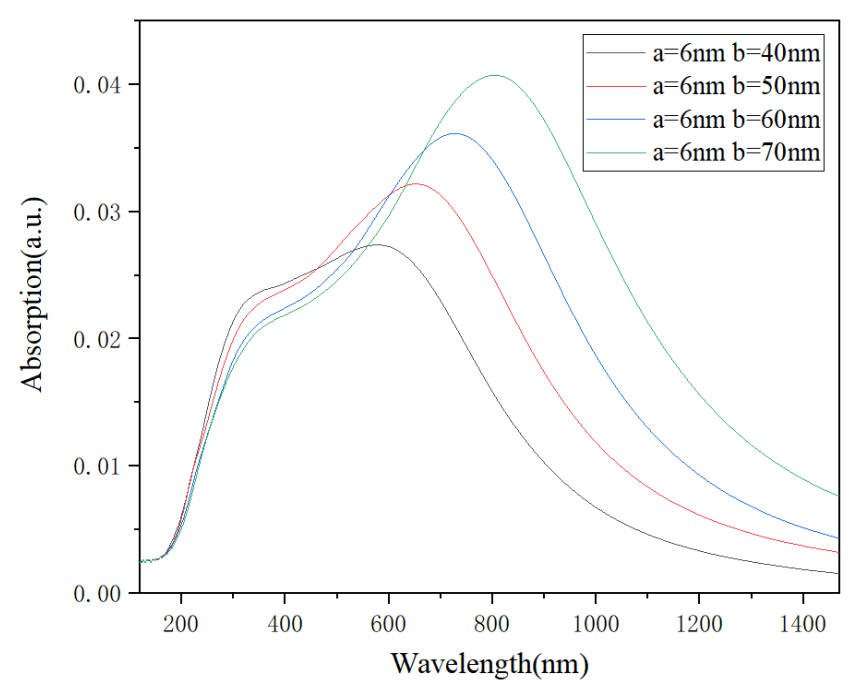

Figure 9. Absorption spectra of palladium nanorods with different axial length and the same radius

To sum up, with the growth in axial length, the transverse and longitudinal absorption peaks of palladium and gold nanorods both red shifted. The fastest red shift was observed in the longitudinal absorption peak of palladium nanorods. Comparing the absorption spectra of the two types of nanorods, the SPR of gold nanorods can be tuned in the visible-near infrared region, while that of palladium nanorods can be tuned in ultraviolet-visible-infrared region. In other words, palladium nanorods are more suitable for broad-spectrum tuning of the SPR.

\subsection{Effects of radius on the SPR of palladium nanospheres and nanorods of the same axial length}

Figure 10 provides the absorption spectra of palladium nanospheres and nanorods with the same axial length $(54 \mathrm{~nm})$ and different radii $(3,6,10$ and $11 \mathrm{~nm})$. As shown in the figure, with the growth in radius, the absorption peaks of palladium nanospheres appeared were blue shifted slightly from $216 \mathrm{~nm}$. For palladium nanorods, the transverse absorption peak was located near $340 \mathrm{~nm}$, while the longitudinal absorption peak changed rapidly from 768 to $479 \mathrm{~nm}$; the longitudinal absorption peak blue shifted faster than transverse absorption peak; the blue shift of the longitudinal absorption peak was accompanied with an obvious decrease of the FWHM. Therefore, palladium nanorods are more effective for broadspectrum SPR tuning than palladium nanospheres.

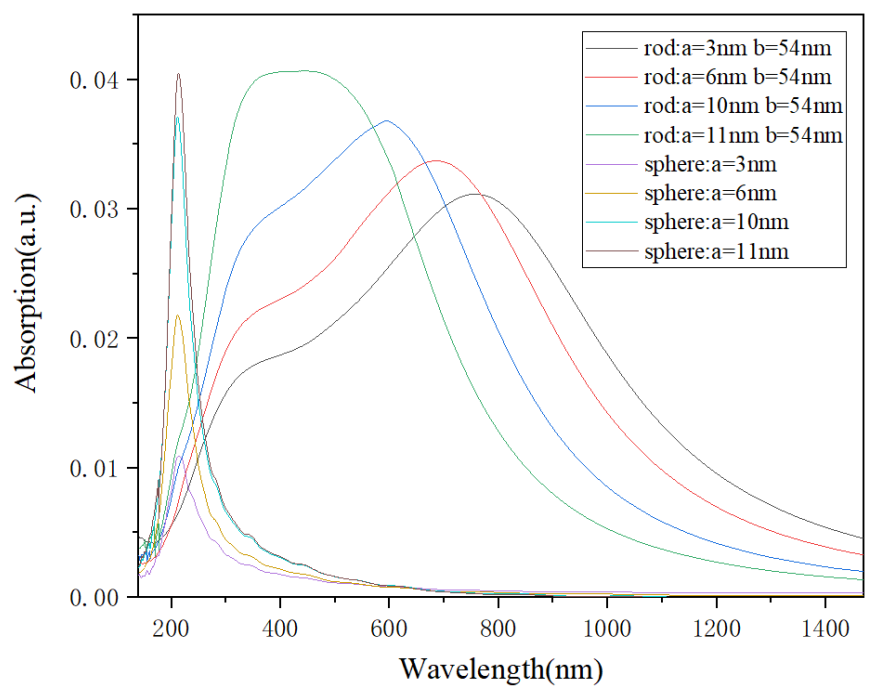

Figure 10. Absorption spectra of palladium nanospheres and nanorods with different radii

3.6 Absorption spectra of palladium nanorods with different sizes with the longitudinal absorption peak at $1,330 \mathrm{~nm}$

Figure 11 gives the absorption spectra of palladium nanorods with different radii $(3,4,5,6,7,8,9,10,11,12$ and $13 \mathrm{~nm})$ and axial lengths $(136,90,150,170,190,200,230$, $220,270,300$ and $310 \mathrm{~nm})$.

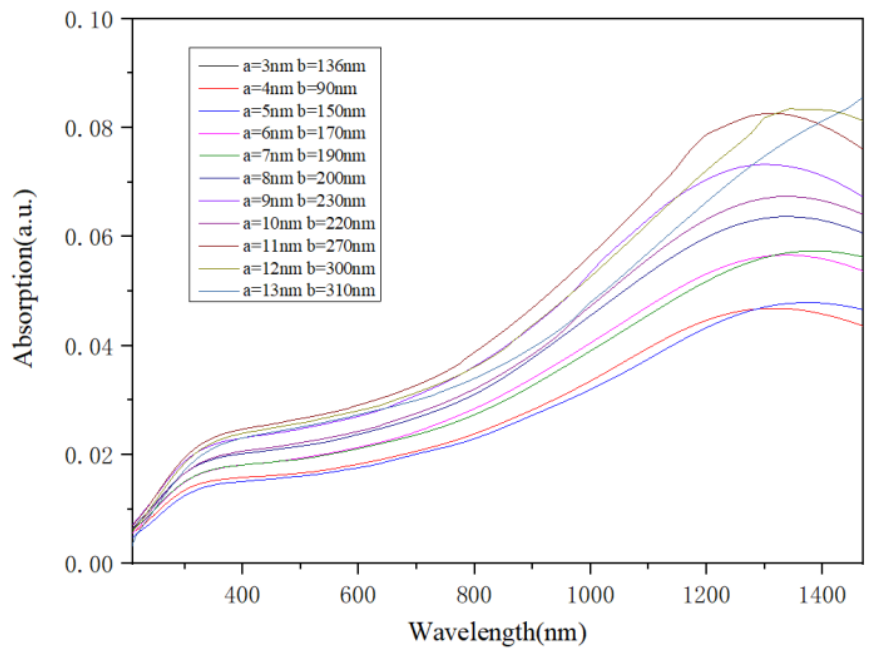

Figure 11. Absorption spectra of palladium nanorods with different sizes with the longitudinal absorption peak at $1,330 \mathrm{~nm}$

As shown in this figure, with the growth in the size of palladium nanorods, the transverse absorption peak had a small blue shift, the longitudinal absorption peak generally fell at $1,330 \mathrm{~nm}$, which is the working wavelength of optical fibers, and the absorption rate showed a rising trend. In other words, when the longitudinal absorption peak remained at the same wavelength, the transverse absorption peak generally had a 
limited blue shift, indicating that the axial length of palladium nanorods need to be increased.

\section{CONCLUSIONS}

(1) With the transverse absorption peak in the ultraviolet region and the longitudinal absorption peak in visible-infrared region, the palladium nanorods have achieved broad-spectrum SPR. Thus, the longitudinal absorption peak of the palladium nanorods can be used to tune the SPR from the visible region to the infrared region.

(2) The SPR of gold nanorods can be tuned in the visiblenear infrared region, while that of palladium nanorods can be tuned in ultraviolet-visible-infrared region, indicating that palladium nanorods are more suitable for broad-spectrum tuning of the SPR.

(3) According to the absorption spectra of palladium nanospheres and nanorods with the same axial length and different radii, palladium nanorods are more effective for broad-spectrum SPR tuning than palladium nanospheres.

(4) The palladium nanoparticles with absorption peaks in the ultraviolet region can be used for up-conversion to kill lesion with ultraviolet; those with absorption peaks in the visible region can be used in photocatalysis, hydrogen production and solar cells; those with absorption peaks in the infrared region can be used to develop biosensors and communication windows.

\section{ACKNOWLEDGMENT}

This work is supported by the Provincial Natural Science Foundation: F2018027.

\section{REFERENCES}

[1] Marvin, W.J. (2003). Handbook of optical materials. CRC Press 331-336. https://doi.org/10.5860/choice.40-4668

[2] Tsai, M.F., Chang, S.G., Cheng, F.Y., Shanmugam, V., Cheng, Y.S., Su, C.H., Yeh, C.S. (2013). Au nanorod design as light-absorber in the first and second biological near-infrared windows for in vivo photothermal therapy. American Chemical Society, 7(6): 5330-5342. https://doi.org/10.1021/nn401187c

[3] Huang, Y.C., Yan, J.H., Ma, C.R., Yang, G.W. (2018). Creating a nanoscale "Black Hole" to trap light by a single $\mathrm{Au}$ nanosphere in an all-dielectric nanocavity. Advanced Optical Materials, 6(20): 1800366. https://doi.org/10.1002/adom.201800366

[4] Codrington, J., Eldabagh, N., Fernando, K., Foley, J.J. (2017). Unique hot carrier distributions from scatteringmediated absorption. ACS Photonics, 4(3): 552-559. https://doi.org/10.1021/acsphotonics.6b00773

[5] Ruan, Q.F., Shao, L., Shu, Y.W., Wang, J.F., Wu, H.K. (2014). Growth of monodisperse gold nanospheres with diameters from $20 \mathrm{~nm}$ to $220 \mathrm{~nm}$ and their core/satellite nanostructures. Advanced Optical Materials, 2(1): 65-73. https://doi.org/10.1002/adom.201300359

[6] Xiong, Y.J., Chen, J.Y., Wiley, B., Xia, Y.N., Yin, Y.D., Li, Z.Y. (2005). Size-dependence of surface plasmon resonance and oxidation for pd nanocubes synthesized via a seed etching process. Nano letters, 5(7): 1237-1242. https://doi.org/10.1021/n10508826

[7] Xiong, Y.J., McLellan, J.M., Chen, J.Y., Yin, Y.D., Li, Z.Y., Xia, Y.N. (2005). Kinetically controlled synthesis of triangular and hexagonal nanoplates of palladium and their Spr/Sers properties. American Chemical Society, 127(48): 17118-17127. https://doi.org/10.1021/ja056498s

[8] Zeng, J., Zhu, C., Tao, J., Jin, M.S., Zhang, H., Li, Z.Y., Zhu, Y.M., Xia, Y.N. (2015). Controlling the nucleation and growth of silver on palladium nanocubes by manipulating the reaction kinetics. Angewandte Chemie International Edition, 51(10): 2354-2358. https://doi.org/10.1002/anie.201108842

[9] Hsu, S.C., Chuang, Y.C., Sneed, B.T., Cullen, D.A., Chiu, T.W., Kuo, C.H. (2016). Turning the halide switch in the synthesis of au-pd alloy and core-shell nanoicosahedra with terraced shells: Performance in electrochemical and plasmon-enhanced catalysis. Nano Letters, 16(9): 55145520. https://doi.org/10.1021/acs.nanolett.6b02005

[10] Rodal-Cedeira, S., Montes-García, V., Polavarapu, L., Solís, D.M., Heidari, H., La Porta, A., Angiola, M., Martucci, A., Taboada, O.M., Obelleiro, F., Bals, S., Pérez-Juste, O., Pastoriza-Santos, I. (2016). Plasmonic $\mathrm{Au} @ \mathrm{Pd}$ nanorods with boosted refractive index susceptibility and SERS efficiency: A multifunctional platform for hydrogen sensing and monitoring of catalytic reactions. Chemistry of Materials, 28(24): 9169-9180.

https://doi.org/10.1021/acs.chemmater.6b04941

[11] Mahmoud, M.A. (2013). Surface-enhanced Raman spectroscopy of double-shell hollow nanoparticles: Electromagnetic and chemical enhancements. Langmuir, 29(21): 6253-6261. https://doi.org/10.1021/la400845z

[12] Mahmoud, M.A. (2014). Plasmon resonance hybridization of gold nanospheres and palladium nanoshells combined in a rattle structure. The Journal of Physical Chemistry Letters, 5(15): 2594-2600. https://doi.org/10.1021/jz501201p

[13] Niu, W., Zhang, W., Firdoz, S., Lu, X.M. (2014). Controlled synthesis of palladium concave nanocubes with sub-10-nanometer edges and corners for tunable plasmonic property. Chemistry of Materials, 26(6): 2180-2186. https://doi.org/10.1021/cm500210u

[14] Du, J.S., Yu, J.J., Xiong, Y.L., Lin, Z.Q., Zhang, H., Yang, D.R. (2014). Developing an aqueous approach for synthesizing $\mathrm{Au}$ and $\mathrm{M} @ \mathrm{Au}(\mathrm{M}=\mathrm{Pd}, \mathrm{CuPt})$ hybrid nanostars with plasmonic properties. Physical Chemistry Chemical Physics, 17(2): 1265-1272. https://doi.org/10.1039/C4CP04757E

[15] Li, Y., Yan, Y.C., Li, Y.H., Zhang, H., Li, D.S., Yang, D.R. (2015). Size-controlled synthesis of Pd nanosheets for tunable plasmonic properties. CrystEngComm, 17: 1833-1838. https://doi.org/10.1039/C4CE02062F

[16] Sugawa, K., Tahara, H., Yamashita, A., Otsuki, J., Sagara, T., Harumoto, T., Yanagida, S. (2015). Refractive index susceptibility of the plasmonic palladium nanoparticle: Potential as the third plasmonic sensing material. ACS Nano, 9(2): 1895-1904. https://doi.org/10.1021/nn506800a

[17] Manchon, D., Lermé, J., Zhang, T., Mosset, A., Jamois, C., Bonnet, C., Rye, J.M., Belarouci, A., Broyer, M., Pellarina, M., Cottancin, E. (2015). Plasmonic coupling with most of the transitionmetals: A new family of broad 
band and near infrared nanoantennas. Nanoscale, 7(3): 1181-1192. https://doi.org/10.1039/c4nr05383d

[18] Verma, P., Kuwahara, Y., Mori, K., Yamashita, H. (2015). Synthesis and characterization of $\mathrm{Pd} / \mathrm{Ag}$ bimetallic nanocatalyst on SBA-15 mesoporous silica as a plasmonic catalyst. Journal of Materials Chemistry A, 3(37): https://doi.org/10.1039/C5TA04818D

[19] Kim, G.W., Lee, S.Y., Ha, J.W. (2017). Threedimensional defocused orientation sensing of single bimetallic core-shell gold nanorods as multifunctional optical probes. The Analyst, 142(6): 899-903. https://doi.org/10.1039/c6an02280d

[20] Wang, Z., Song, Y., Zou, J., Li, L.Y., Yu, Y., Wu, L. (2017). The cooperation effect in the $\mathrm{Au}-\mathrm{Pd} / \mathrm{LDH}$ for promoting photocatalytic selective oxidation of benzyl alcohol. Catalysis Science \& Technology, 8(1): 268-275. https://doi.org/10.1039/C7CY02006F

[21] Nugroho, F.A.A., Darmadi, I., Zhdanov, V.P., Langhammer, C. (2018). Universal scaling and design rules of hydrogen-induced optical properties in $\mathrm{Pd}$ and Pd-alloy nanoparticles. ACS Nano, 12(10): 9903-9912. https://doi.org/10.1021/acsnano.8b02835

[22] Wang, S.M., Fu, J.W., Wang, K., Gao, M., Wang, X.Z., Wang, Z.W., Chen, J.F., Xu, Q. (2018). Facile synthesis of $\mathrm{Pd}$ nanoparticles on polydopamine -coated $\mathrm{Fe}-\mathrm{Fe}_{2} \mathrm{O}_{3}$ magnetic nanochains as recyclable high-performance nanocatalysts. Applied Surface Science, 459: 208-216. https://doi.org/10.1016/j.apsusc.2018.07.205

[23] Watkins, W.L., Yves, B. (2018). Ultrasensitive and fast single wavelength plasmonic hydrogen sensing with anisotropic nanostructured $\mathrm{Pd}$ films. Sensors and Actuators B: Chemical, 273: 527-535. https://doi.org/10.1016/j.snb.2018.06.013

[24] Xu, C.Y., Huang, W.H., Li, Z., Deng, B.W., Zhang, Y.W., Ni, M.J., Cen, K.F. (2018). Photothermal coupling factor achieving $\mathrm{CO}_{2}$ reduction based on palladiumnanoparticle-loaded $\mathrm{TiO}_{2}$. ACS Catalysis, 8(7): 65826593. https://doi.org/10.1021/acscatal.8b00272 